\title{
The simulation of canopy fabric air permeability's influence on the round parachute during the landing process
}

\author{
Ma Yunpeng ${ }^{1, a}$, Zu Jinge ${ }^{2, b}$ \\ ${ }^{1}$ School of Aeronautic Science and Engineering, Beihang University, Beijing, 100191, China \\ ${ }^{2}$ School of Aeronautic Science and Engineering, Beihang University, Beijing, 100191, China \\ amyp@buaa.edu.cn, ${ }^{\mathrm{b}}$ zujinge@emast.com.cn
}

Keywords: Round parachutes, fabric air permeability, steady decline stage, simulation research.

\begin{abstract}
Since the parachute descent is more complex, the cost dropped and other tests designed to be very large, simulation studies with a very large parachute design significance.Parachute canopy breathable fabric has great influence on the parachute descent, The purpose of this study is to consider the case of breathable fabric, parachute simulation study to explore and study the impact of the round parachute fabric breathability steady decline stage, breathable fabric will parachute into account is a major feature of the simulation. The simulation results show the feasibility of this article parachute simulation research methods, and by the results of comparative analysis, found breathable fabric canopy has an important effect on the parachute drop after full phase. As appropriate to improve fabric permeability, can reduce the drag coefficient, reducing the pressure difference between the upper and lower surface of the canopy, reduce flow separation vortex phenomenon, increasing the stability of the parachute.
\end{abstract}

\section{Introduction}

With the parachute in the field of aerospace and other military as well as civilian areas with a wide, parachute simulation study of technology has also been a great development. The use of CFD software and finite element knowledge, parachute to simulate become the main method of parachute simulation study. The main parachute simulation is fluid-structure interaction problems, but because of the canopy fabric breathable, so the simulation is different from the general simulation model.This article aims to explore the issues to consider simulation parachute canopy fabric breathability case study the effect of the permeability of fabric canopy parachute drop process. The simulation will consider the problem of the simulation in case of air-permeable fabric becomes complicated parachute drop each stage, a steady decline in the shape of the parachute relative to the fixed stage, the simulation model is relatively simple. So this paper selected round parachute model, using FLUENT software, set a different fabric breathability, simulate its steady decline stage, exploring the impact of the round parachute fabric breathability steady decline stage through simulation comparison. Simulation of the flow Fig.1 shown below:

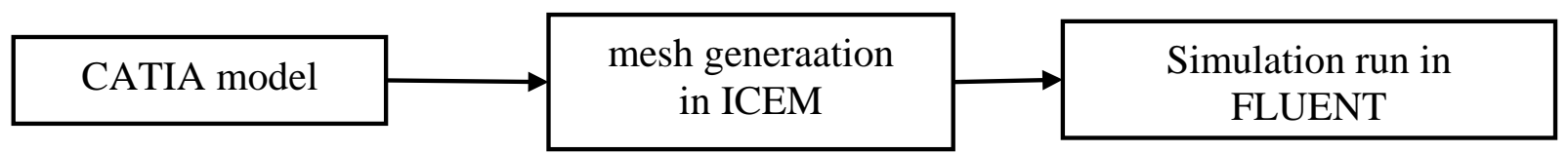

Fig.1 Simulation flowchart

\section{Simulation Model}

Physical Model. Parachute model based on C9 parachute, C9 parachute is an open top hole round parachute. Taking into account the C9 parachute model is the center of symmetry and axial symmetry model, one-half of the entire parachute models also take into account the computing power of computers, and the simulation is more simple and quick to make, a decision establishing a 
model only half the parachute model, using simulation calculation. Parachute half model shown in Fig 2.

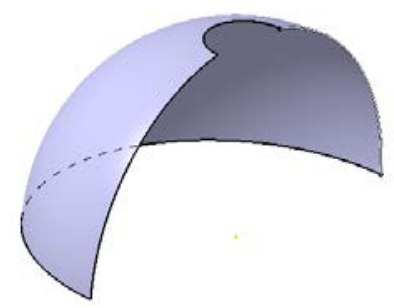

Fig.2 Parachute model

Research is mainly stable parachute descent, so the simulation of the flow field is a bottom-up from the bottom of the gas flow field parachute.Establishment of the flow field shown in Fig.3.

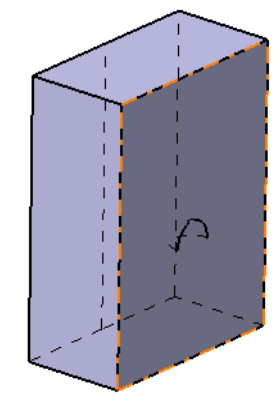

Fig.3 Flow model

Mathematical Model. Equations using the N-S equation. Parachute is a general flow around the flow of an incompressible fluid, without solving the energy conservation equation, solving the basic equations of flow around a parachute flow problems require the following:

Mass conservation equations(Continuity equation):

$\frac{\partial}{\partial x_{i}}\left(\rho u_{i}\right)=0$

Taking into account the steady decline stage parachute flow field can be seen as an approximate flow around a blunt body movement, Canopy interior is remarkable turbulent flow, canopy above the tail will produce significant flow separation, vortex. Because the flow of non-steady-state canopy with spin irregular flow, further integration of the two partial differential equations N-S equations, thus parachute simulation study on mathematical models to select k- $\varepsilon$ model is widely used on fluid simulation.

Boundary Conditions. Solid-wall boundary conditions (wall) on the circle parachute surface, ie no penetration and thermal insulation and no-slip boundary; outside the boundaries of the computational domain and flow rate using the import and export of boundary conditions boundary conditions for incompressible gas flow simulation .The breathable fabric canopy, using a porous medium model simulations, you can use the porous medium boundary conditions due to the canopy surface as a film, and thus calculate the permeability (Porous jump).

Pressure changes in the porous medium boundary conditions given by the following formula:

$$
\Delta p=\left(\frac{\mu}{C_{1}} \cdot \lambda+C_{2} \cdot \frac{1}{2} \cdot \rho \cdot \lambda^{2}\right) \cdot \delta
$$

Paper simulates the permeability of the fabric under three conditions, a stable descent circular parachute. Permeability from small to large order of 602 Cotton yarn 66 Silk, 109 Cotton, 509 brocade. The parameter settings in the following Table1: 
Table 1 parachute fabric two parameters

\begin{tabular}{|c|c|c|}
\hline & $C_{1} \times 10^{11}\left(\mathrm{~m}^{2}\right)$ & $C_{2} \times 10^{-5}(1 / \mathrm{m})$ \\
\hline 602 brocade 66 silk & 4.589 & 10.95 \\
\hline 509 brocade & 7.942 & 3.026 \\
\hline 109 cotton & 3.602 & 1.631 \\
\hline
\end{tabular}

\section{The simulation results}

Circular parachute symmetry Streamline diagrams show as Fig.4

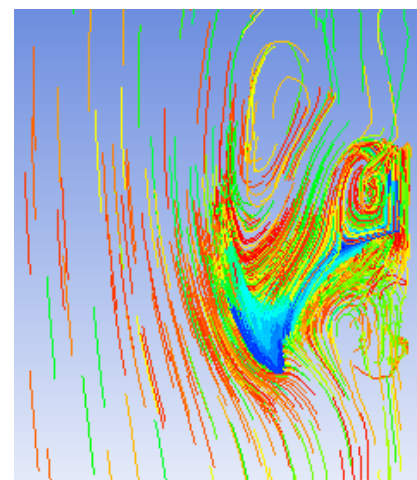

(a)602 brocade 66 silk

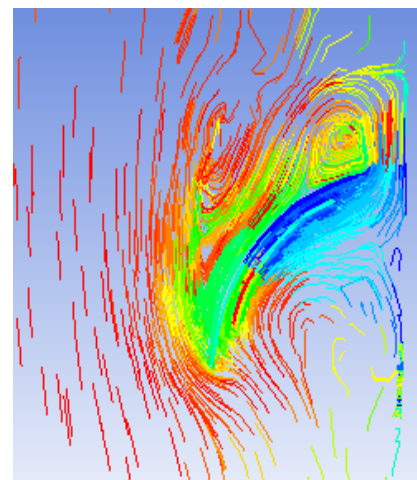

(b)509 brocade

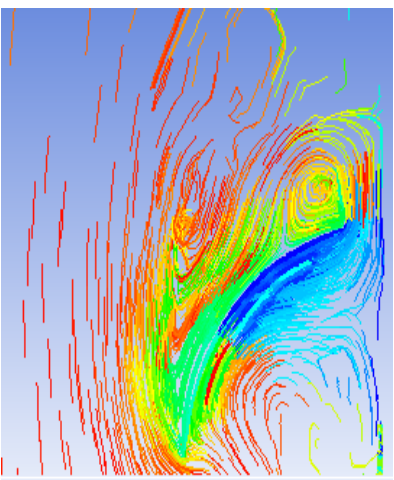

(c)109cotton

Fig.4 Streamline diagrams

Circular parachute symmetry plane pressure. Open-top round-pressure cloud parachute symmetry plane as shown Fig.5:

Analysis of the in-plane symmetry flow chart we can find: when the air flow near by the round parachute, there are vortex in the top surface of the parachute, resulting flow separation. And comparison can be found with the canopy fabric permeability increase, decrease air swirl become obvious, which can determine the stability of the parachute is enhanced.

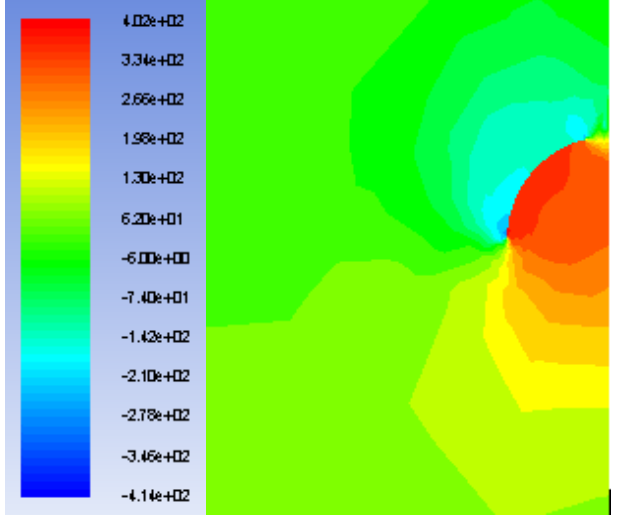

(a)602 brocade 66 silk

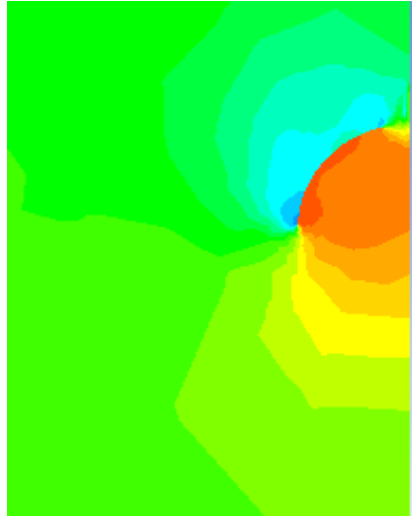

(b)509 brocade

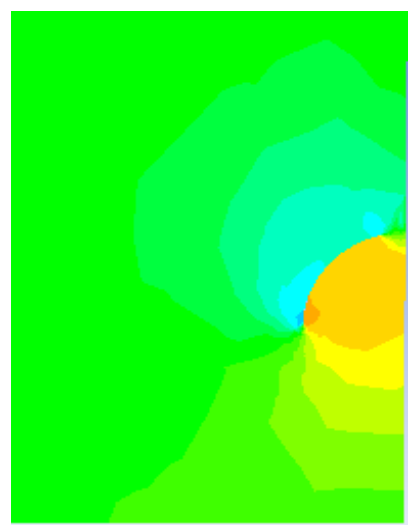

(c)109cotton

Fig.5 Stress nephogram 
It can be found that the pressure of canopy's interior is larger. The top surface's pressure of canopy is less, even form subatmospheric pressure, because of the burbling flow spreation. It generate a differential pressure between the top and bottom of canopy. With the increasing of fabric canopy's air permeabilty, the differential pressure is decreasing.

\section{Conclusion}

In this paper, porous media model in FLUENT well round off the top of the parachute steady decline stages of simulation studies, feasibility studies have shown that the use of porous media simulation parachute fabric breathability, and concurrency are within a certain range, increasing the open top Kongyuan parachute clothing fabric air permeability can be reduced near the upper and lower surface of the canopy pressure, reduced by the bevel top opening air flow rate to reduce the air flow the phenomenon analysis and increase its stability.

\section{References}

[1] Wang Lirong, Theory and application of parachute[M]. BeiJing: China Astronautic Publishing House,1997.

[2] Ma YanFu.Parachute fabric air permeability . 26-30.

[3] YuLi, MingXiao, Chen Lijun. Experimental study on the flow field of different vent parachute

[J]. Experimental study on the flow field of different vent parachute, 2008, 26(1), 19-24.

[4] Yu Hongxuan.Research on fluid structuer integration of the parachute on opening processing [D]. Nanjing University of Aeronautics and Astronautics, 2010.

[5] Liu Wei, Kou Baohua, Ge Jianquan, Yang Tao, Zhang Xiaojin. Numerical simulation of flow field with stable stage of parachute[J]. Chinese space since and technology. 2007, 1, 61-64. 